\title{
A New Methodology for RF MEMS Simulation
}

\author{
Peyrou David, Coccetti Fabio, Achkar Hikmat, Pennec Fabienne, \\ Pons Patrick and Plana Robert \\ LAAS-CNRS, Toulouse University \\ France
}

\section{Introduction}

RF MEMS have demonstrated, during the last ten years, very attractive potential to allow the introduction of "intelligence" in the RF front end architecture (Rebeiz \& Muldavin, 2001) through analog signal processing techniques. Nevertheless, those devices with moveable structures still have some issues to be successfully introduced at the industrial level.

The first issue deals with the actuation medium and the corresponding reliability. Today, it is well known that membranes and cantilevers can be actuated through electrostatic, thermal, magnetic and piezoelectric forces. Each of these types of actuators features benefits and drawback and it seems that today electrostatic actuators could offer the best trade-off when issues concerning the very high actuation voltage and reliability issues are solved (Mellé et al., 2005) (Yuan et al., 2006) (Reid \& Webster, 2002) (Sprengen et al., 2004). As they are made of moveable and fragile structures (beams, cantilevers...), RF MEMS switches must be encapsulated in order to protect them from hostile environments, to increase their reliability and lifetime.

The second issue comes from the lake of efficient and easy-to-use simulation tools covering the complete MEMS design procedure, from individual MEMS component design to complete system simulation. Finite element analysis (FEA) methods offer high efficiency and are widely used to model and simulate the behaviour of MEMS components.

However, as MEMS are subject to multiple coupled physical phenomena (Figure 1) at process level and play, such as initial stress, mechanical contact, temperature, thermoelastic, electromagnetic effects, thus finite element models may involve large numbers of degrees of freedom so that full simulation can be prohibitively time consuming. As a consequence, designers must simplify models or specify interesting results in order to obtain accurate but fast solution.

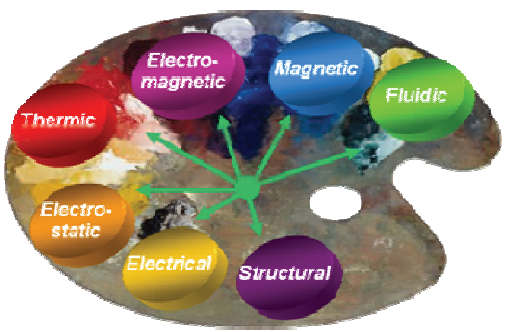

Figure 1. MEMS are multiphysics 
In this problematic, we are working on a systematic and robust conception flow. Figure 2 describes the whole conception flow, starting from the conceptual specifications thru mask design and finishing with the complete simulation at system level.

The main challenge concerns the bottom-up conception flow (Figure 2) which needs a special effort in the modeling of the full process of MEMS technology. In fact we need to create a virtual prototype of the device able to take into account the inherent interrelated physical phenomena at process level and play, such as initial stress, mechanical contact, temperature, thermoelastic, electromagnetic effects.

The best way to solve each coupled physics simultaneously (not sequentially) relies on using only one tool. For example, two softwares provided by ANSYS and COMSOL offer multiphysics environment. Even these multiphysics software are great, we cannot hope a well proven and dedicated tool to solve each specifics physics with respect to a reasonable time consuming. So this chapter outlines an original approach based on reverse engineering method used to both interface different Computer-Aided Design (CAD) softwares each other and also advanced characterization software.

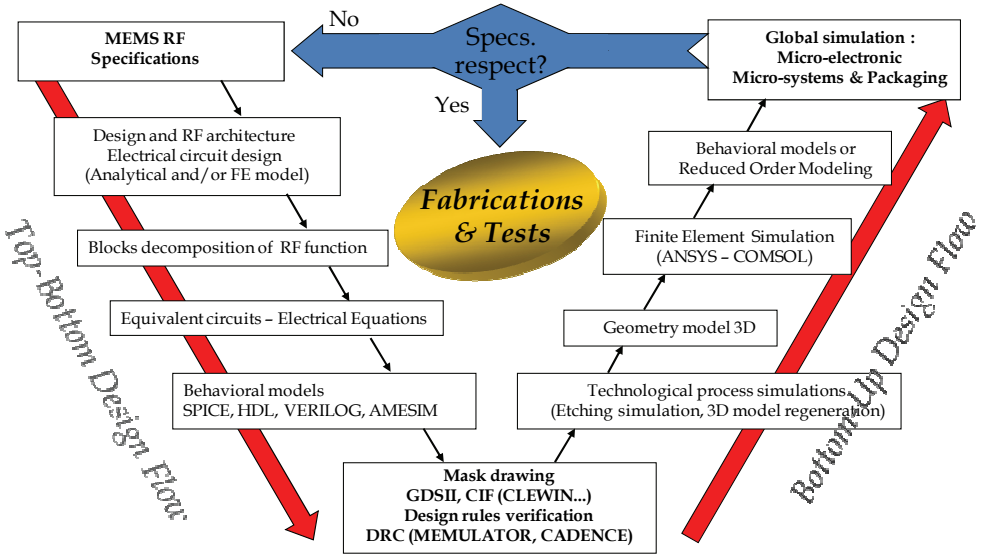

Figure 2. Systematic and robust conception flow

\section{Reverse Engineering method}

The basic idea is really simple, reverse engineering is a method to create a $3 \mathrm{D}$ virtual model of an existing physical part for use in 3D CAD software. The reverse engineering process involves measuring an object and then reconstructing it as a 3D model. The physical object can be measured using 3D scanning technologies like optical profilometer or Atomic Force Microscopy (AFM) for example. The measured data alone, usually represented as a point cloud, lacks topological information and is therefore processed and modeled into a more usable format such as a stereolithography format which is an ASCII file used in standard CAD softwares.

Thus, our concept relies on this method to first build the model using the real shape of the device (process level) and then to interface different software each other. The last point needs to create a new 3D virtual model from the deformed shape obtained with the simulations made by the software called 1 as the input of software called 2, so a sequential coupling can be done using different software. 


\section{Applications}

\subsection{Capacitance simulation}

This application shows our reverse engineering method to discover the origin of the mismatch between the theoretical down state capacitance and measurements of an RFMEMS Capacitive Switch. This numeric approach allows us to understand the roughness effect of the dielectric on the down-state capacitance. The agreement between the modelling and the analytical model is very good and validates this novel numeric step.

For capacitive switches, both dielectric charging and surface roughness (Palasantzas \& DeHosson, 2006) will impact the isolation. So, to enhance the down-state capacitance, we focus our work on a methodology to simulate the real capacitance using the measured data of the dielectric surface. The goal of this application is to show how to set up and generate a CAD model obtained by measured data.

\subsubsection{Roughness characterization}

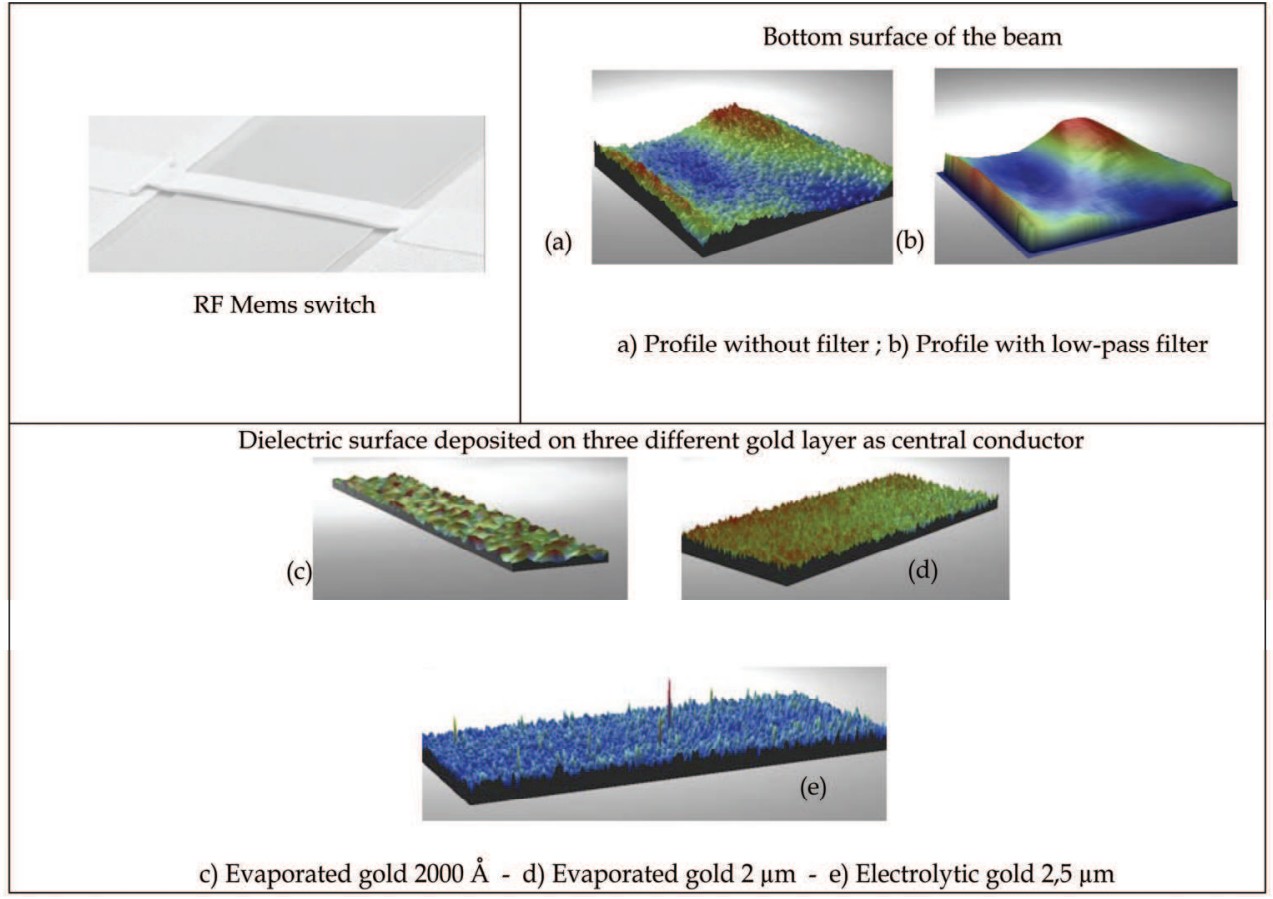

Figure 3. Roughness characterization for RF MEMS switch

Topographical characterization of both the textured dielectric on the central conductor above the beam and the gold surface on the bottom was performed using optical profilometry (Figure 3). This technique, using Wyko NT1000 from Veeko instrumentation, allows to map the surface in 3-D without affecting the surface properties or deforming the substrate. Profilometry data and 3-D rendering of the surface was accomplished using WYKO Vision32 version 2.303 software package. 
Many parameters are used to describe surface roughness but we can distinguished two main parameters (see Figure 4):

- $\quad \mathrm{Ra}$, Roughness Average (Absolute value of the surface height averaged over the surface ie high frequency aspect)

- la, Average Wavelength (mean spacing between peaks, with a peak defined relative to the mean line ie low frequency aspect)

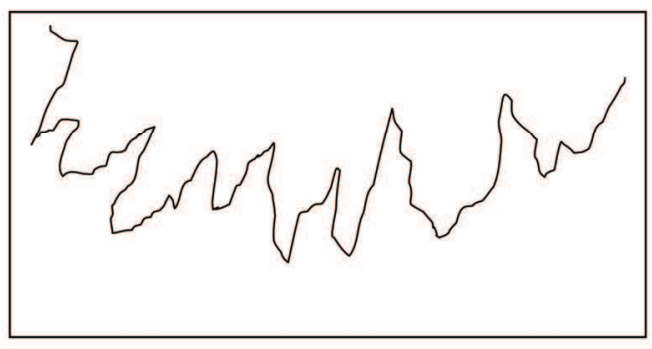

Real profile of the probed surface

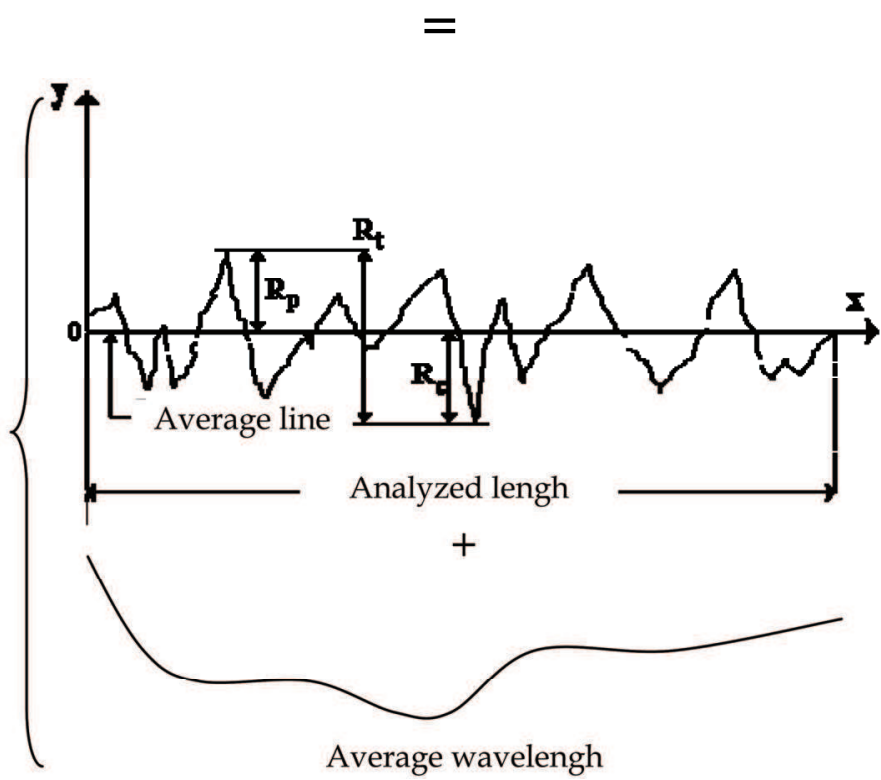

Figure 4. Roughness decomposition

\subsubsection{Analytic expression of the down state capacitance}

We define an analytic model for the down state capacitance of RF MEMS Switch (see Figure 5) which take into account air gap between the beam and the dielectric using low-pass filter (average wavelength) and high-pass filter (average roughness) as it is shown in figure 3. 


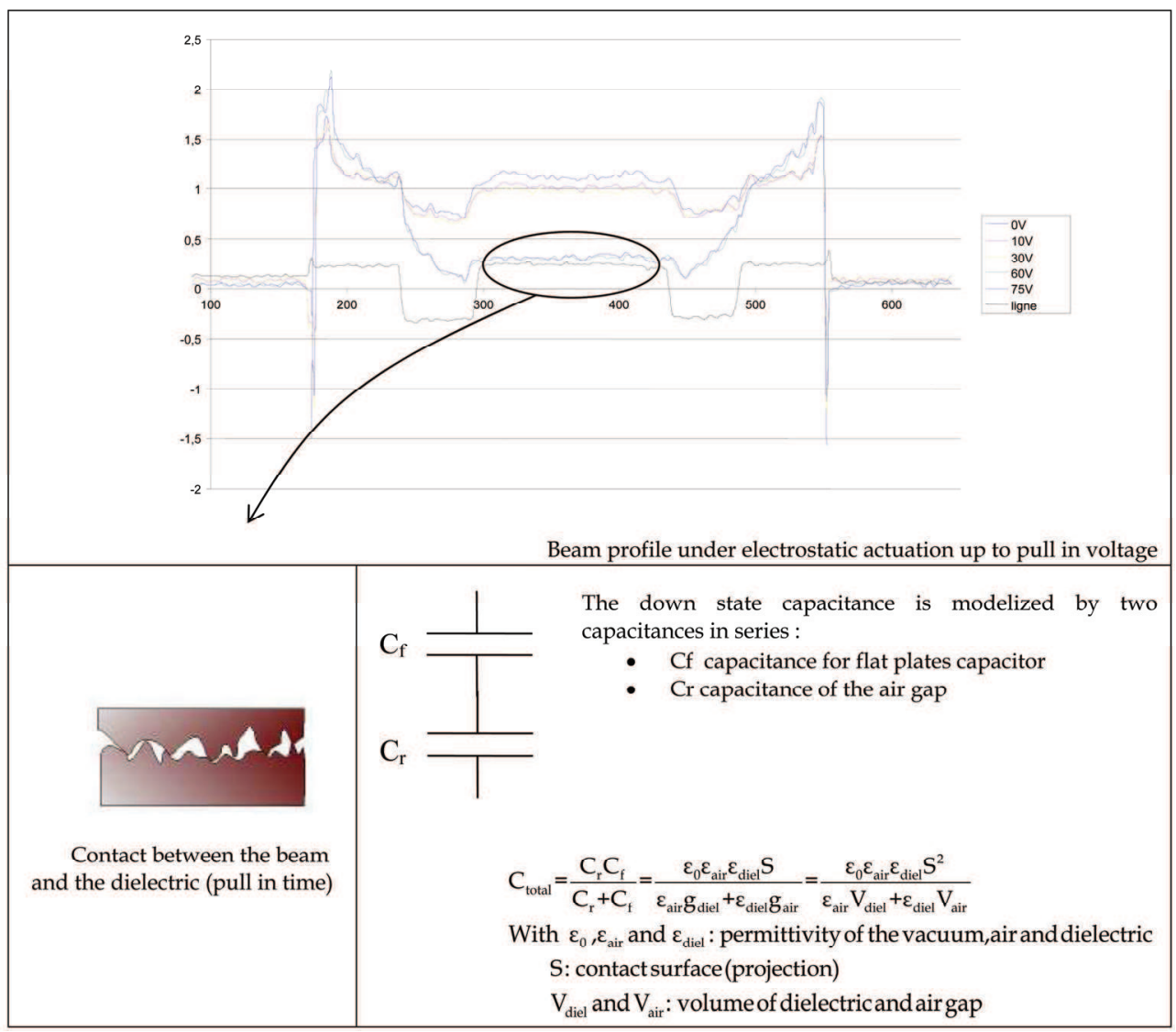

Figure 5. Capacitance model

\subsubsection{Reverse engineering methodology}

We used an optical profilometer (VEECO) to capture three-dimensional (3D) data points of the dielectric surface. Then, using Matlab functions we insert the generated surface as the top surface of a block. For CAD modeling, the segmented data are further transformed into individual surfaces. Several mathematical schemes for representing geometrical shapes are available. We choose to convert the closed surface into stereolithography format because this is an ASCII file used in most standard CAD softwares. Moreover, generated solid model are described by a list of the triangular surfaces which can be easily implemented on Matlab. The last step is to import the generated solid into a CAD software (COMSOL) to make the down-state capacitance analysis.

Figure 6 illustrates the full method and data flow : dielectric roughness is scanned by the optical profilometer (A) to generate an ASCII file (B) representative of 3D coordinates points of the surface. Than solid model (C) and object file (E) as STL, VRML or Comsol geometry can be obtained using Matlab. To enhance the shape, one can use (D) some filters (filter2 for MatLab or a CAD software, CATIA for example). 
After this, the model is ready to be imported into a finite element software (F). We used multiphysics software provided by COMSOL, to set up the electrostatic model (materials and boundaries conditions $(\mathrm{F})$ - meshing $(\mathrm{G})$, results and post processing $(\mathrm{H})$ ).
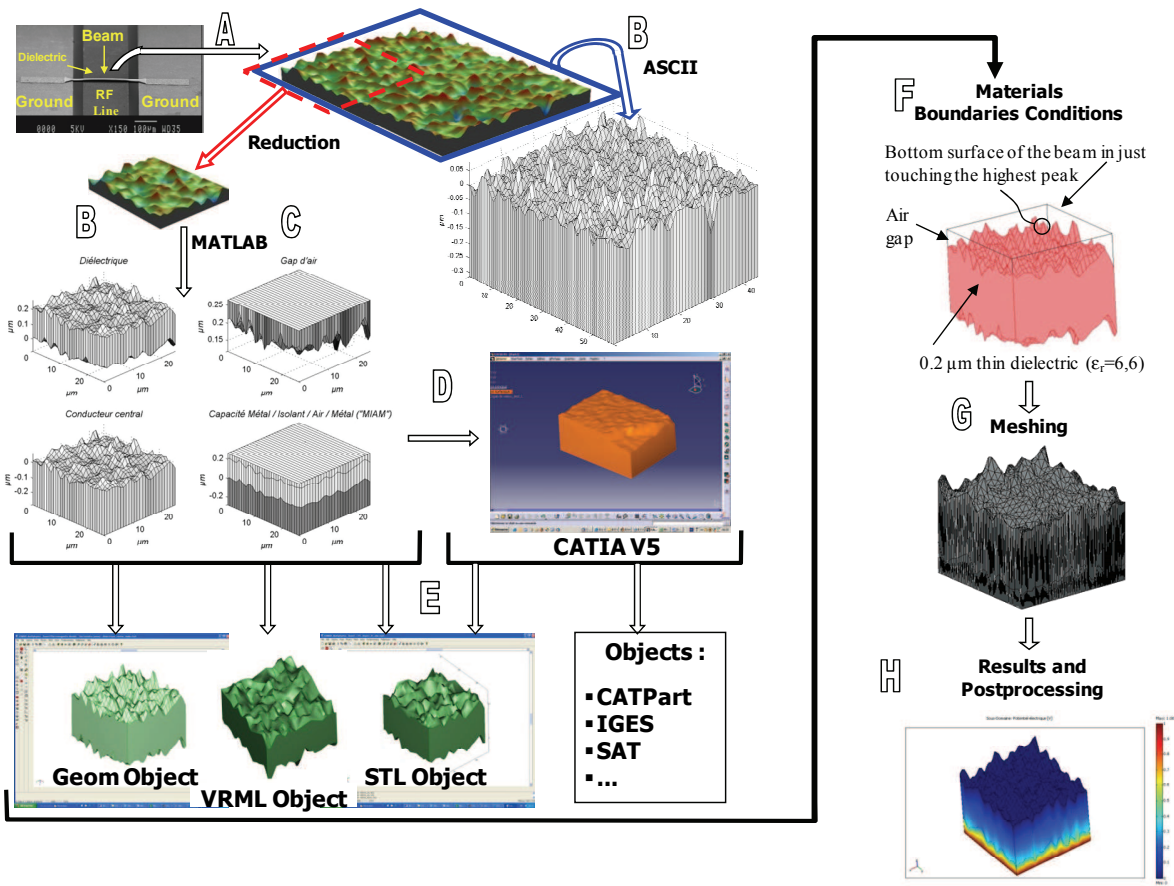

Figure 6. Description of the Reverse Engineering method

\subsubsection{Results and discussions}

Measured capacitances were realized using automatic probe station facility (Karl Suss AP6, see Figure 7) including an optical profilometer (Fogale).

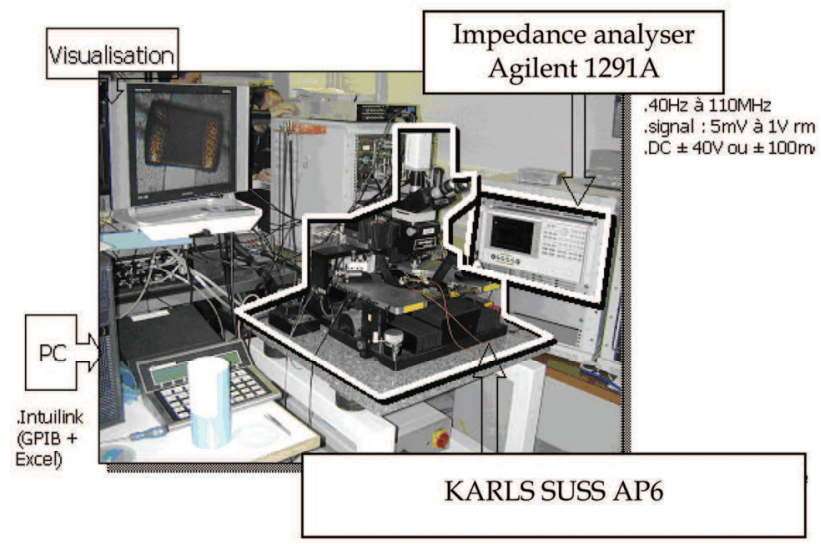

Figure 7. Capacitance bench test 
In this section, we present results about measured capacitance at down state with the following dimensions and materials properties:

- Central conductor : Thick electrolytic gold $(2.5 \mu \mathrm{m})$

- $\quad$ Dielectric : Thin layer $(0.2 \mu \mathrm{m})$, permittivity of 6.6

- The surface area related to the flat plate area is $130 \times 80 \mu \mathrm{m}^{2}=1.04 \mathrm{e} 4 \mu \mathrm{m}^{2}$

Table 1 shows measurements realized with the capacitance bench test on five samples, the mean capacitance is about $1,41 \mathrm{pF}$.

\begin{tabular}{|c|c|}
\hline Sample number & Measured capacitance \\
\hline 1 & $1,60 \mathrm{pF}$ \\
\hline 2 & $1,35 \mathrm{pF}$ \\
\hline 3 & $1,36 \mathrm{pF}$ \\
\hline 4 & $1,29 \mathrm{pF}$ \\
\hline 5 & $1,46 \mathrm{pF}$ \\
\hline Mean capacitance & $1,41 \mathrm{pF}$ \\
\hline
\end{tabular}

Table 1. Measured capacitance for five samples

In order to decrease the number of freedom during the finite element analysis, capacitance simulations were performed using a reduced area $\left(647.61 \mu \mathrm{m}^{2}\right)$ mapped by the optical profilometer. So we assume the roughness fluctuations of the in plane position is correctly estimated by the reduced sample. Thus we modified the simulated capacitance multiplying it by 16 which is the ratio of the real area and the simulated area $(1.04 \mathrm{e} 4 / 647.61=16)$.

Simulated capacitance is obtained using a multiphysics software (COMSOL 3.2). Table 2 shows the simulated and corrected results obtained from simulation as shown in Figure 8.

\begin{tabular}{|c|c|}
\hline Simulated capacitance & $\begin{array}{c}\text { Simulated capacitance } \\
\text { with area correction }(x 16)\end{array}$ \\
\hline $6,222879 \mathrm{e}-2 \mathrm{pF}$ & $0.999335 \mathrm{pF}$ \\
\hline
\end{tabular}

Table 2. Simulated capacitance

Equation 1 gives the analytic evaluation of the down state capacitance (expression shown before in Figure 4) uses air gap and dielectric volumes obtained by integration during simulations (see Figure 7) :

$$
\mathrm{C}_{\text {total }}=\frac{\varepsilon_{0} \varepsilon_{\text {air }} \varepsilon_{\text {diel }} S^{2}}{\varepsilon_{\text {air }} V_{\text {diel }}+\varepsilon_{\text {diel }} V_{\text {air }}}=0.9571255 \mathrm{p} \mathrm{F}
$$


To show the influence of the roughness we calculated the theoretical capacitance (Eq.2) for flat plate capacitor:

$$
\mathrm{C}_{\mathrm{th}}=\frac{\varepsilon_{0} \varepsilon_{\mathrm{diel}} \mathrm{S}}{\mathrm{g}_{\text {air }}}=3.034554 \mathrm{pF}
$$

As shown in table 3, simulated and calculated capacitances are closed within $4.2 \%$. This difference could be explained by the electric field gradient near the peaks that increased locally the capacitance, theses effects are not introduced in our analytic model.

\begin{tabular}{|c|c|c|}
\hline Simulated & Calculated & Measured \\
\hline $0.999335 \mathrm{pF}$ & $0.9571255 \mathrm{pF}$ & $1.41 \mathrm{pF}$ \\
\hline
\end{tabular}

Table 3. Capacitance results

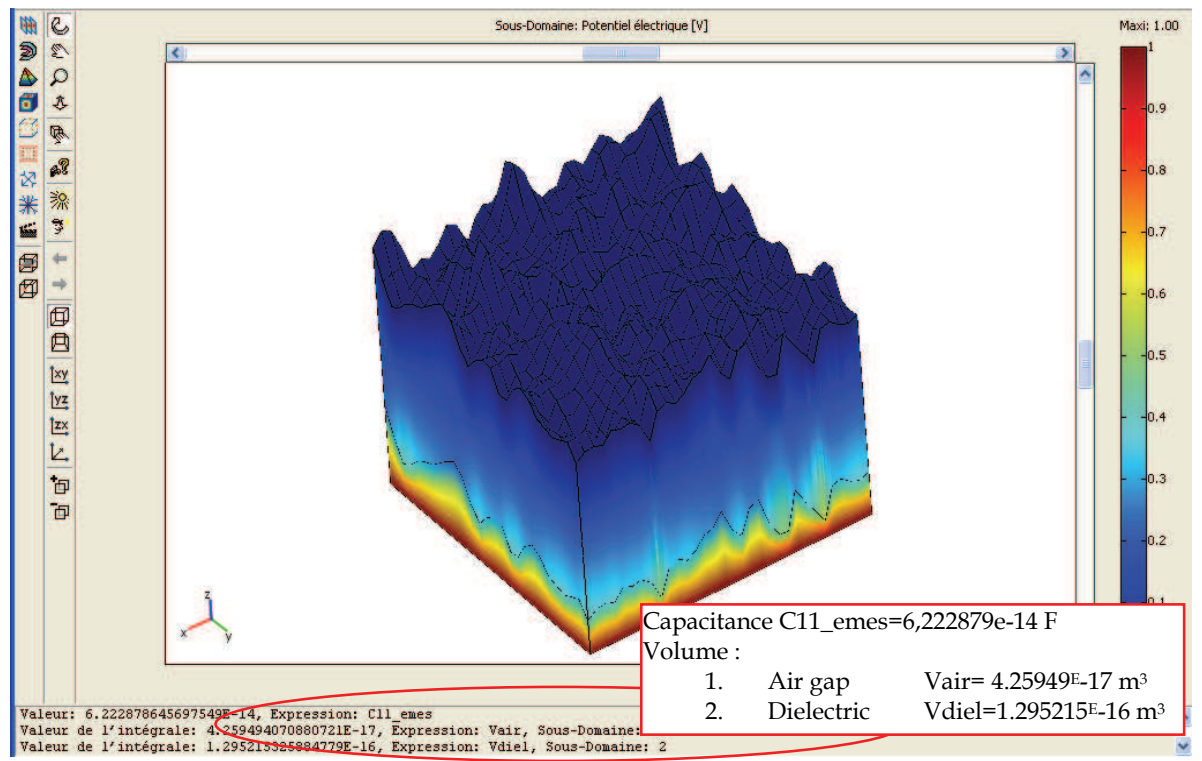

Figure 8. Simulated capacitance (bottom corner: results, Subdomain : Electric field)

But there is an important mismatch, near 30\%, between simulated (or calculated) and measured data of down state capacitance. We assume this difference relies on the three following points:

Simulations were done without accounting:

1. for fringing fields (beam length was taken as the central conductor width)

2. for rough surface of the beam

3. for real contact beam versus dielectric at pull in voltage (we assume the beam to be stopped by the highest dielectric peaks, see Figure 9) 


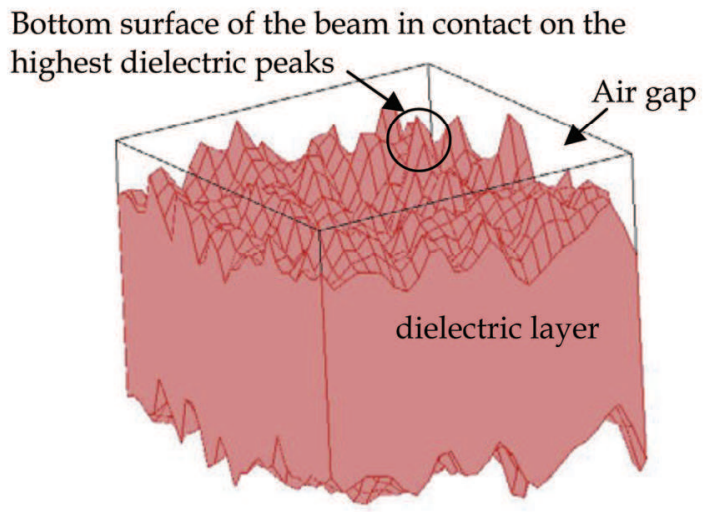

Figure 9. Contact modeling

Simulations show (as predicted) down-state capacitance decrease with the roughness of the dielectric about three time less than the theoretical formulation on flat plate capacitor. We find an accurate analytic model of the down state capacitance which take into account the roughness including an air gap capacitor. Moreover, Mismatches between simulated and measured data are explained and will be studied in further work to improve our model.

\subsection{Electrical contact resistance simulation}

For the DC contact RF MEMS, it has been identified that most of the limitations are related to the quality and the repeatability of the contact that drive the RF performance (insertion losses, isolation, power handling) and the reliability (Rebeiz, 2003). In order to propose new generation of RF MEMS devices, it is important to get a deeper insight on the physic of contact in order to choose appropriate materials, topology and architecture. It has to be furthermore outlined that the insertion of RF MEMS into real architecture will necessitate reduced actuation voltage, dimensions and a better control of the electrical and electromechanical behavior that will give more importance to surface effects.

The testing and development of contact material or contact topology can be addressed with a dedicated experimental set up for monitoring test structures. However, it is difficult to perform the tests under realistic conditions and in particular to duplicate the switch geometry, the contact geometry and the contact force. Moreover, the fabrication process must be optimized and it may take many months to fabricate a set of switches to test a single candidate contact material or contact bump shape.

In order to tackle these issues advanced simulation tools are needed. These tools for finite element analysis allow us to model assembly structures quickly and accurately with a minimal amount of effort. Then, they offer precious guidelines to choose appropriate design parameters and contact material.

In this section, we present an innovative numerical method used to calculate the contact resistance between rough surfaces from real shape of the surfaces that come in contact.

\subsubsection{Reverse engineering methodology}

So far, surface effects were ignored in the analysis, because of the difficulty to generate a rough surface model and also to simplify the model in order to reduce computation times. 
With the increase of computation capabilities, the topography of the surface can be included in finite element simulations. Thus we describe the new methodology that allows the simulation of the DC contact of RF MEMS devices through finite element multi-physics simulation and surface characterization.

A variety of methods to generate rough surfaces have been proposed. But all of these methods deal with a statistical or fractal description of the surface's roughness. The originality of this work relies on a novel approach using a reverse engineering method to generate the real shape of the surface. For this purpose, we used either an optical profilometer (VEECO) or an Atomic Force Microscope (AFM) to capture three-dimensional data points of contact surfaces. Then, using Matlab functions we convert the closed surface from a stereo-lithographic format to an ASCII file compatible with ANSYS Parametric Design Language (APDL). In the final step, the rough surface was obtained by creating key points from the imported file. Since the key points are not co-planar, ANSYS uses Coons patches to generate the surface, and then we used a bottom up solid modeling to create the block volume with the rough surface on the top.

Figure 10 describes the full method developed on ANSYS platform.
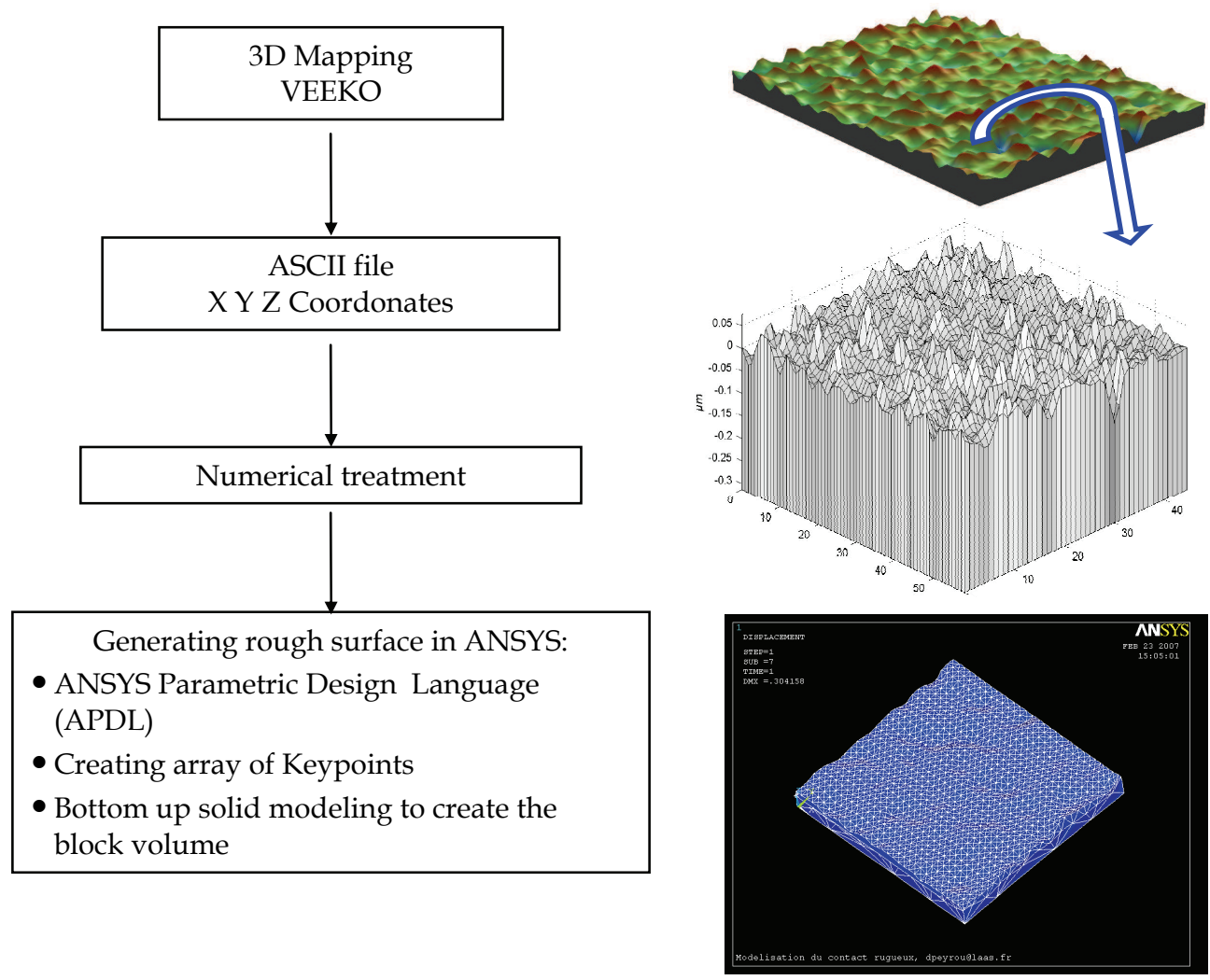

Figure 10. Reverse engineering methodology 


\subsubsection{Model definition and simulation}

The model consists of an electroplated gold layer defined as a flexible material (Figure 11) with a Young's modulus and a Poisson's ratio taken respectively to 80GPa and 0.42 . Yield stress is also introduced and the material behavior is described by a bilinear stress-strain curve starting at the origin with positive stress and strain values. Since the evaporated/electroplated gold bilayer of the beam is deposited during the fabrication on a photoresist sacrificial layer, the roughness of the bottom membrane (dimple) can be neglected. And so the target surface (indentor) is assumed to be a flat, smooth surface.

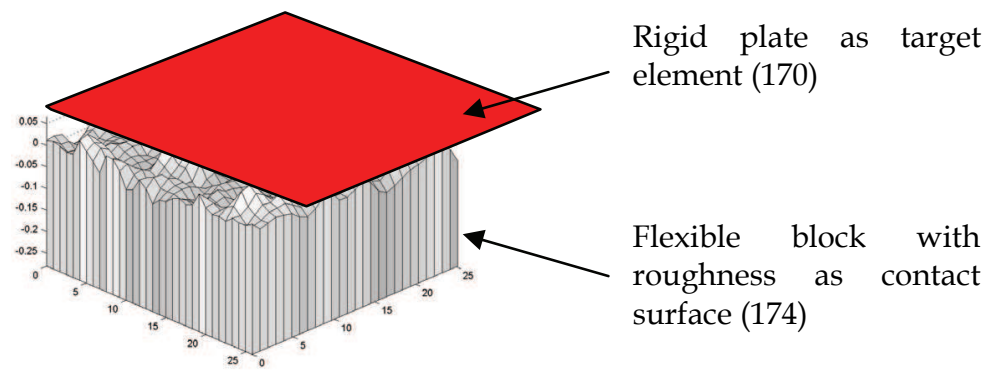

Figure 11. Model definition

To perform the finite element analysis, we choose the combined method based on penalty and lagrangian methods called the Augmented Lagrange method (see Figure 12). That is a penalty method with penetration control :

- The Newton-Raphson iterations start off similar to the pure penalty method.

- Similar to the pure Lagrange multiplier method, the real constant TOLN determines the maximum allowable penetration.

- If the penetration at a given equilibrium iteration exceeds this maximum allowable penetration $\left(\mathrm{x}_{\text {pene }}\right)$, the contact stiffness per contact element $\left(\mathrm{k}_{\text {cont }}\right)$ is augmented with Lagrange multipliers for contact force $\left(\lambda_{\mathrm{i}}\right)$. For the contact element's stiffness, the force is:

$$
\lambda_{\mathrm{i}+1}=\lambda_{\mathrm{i}}+\mathrm{k}_{\text {cont }} \cdot \mathrm{x}_{\text {pene }}
$$

if the penetration is greater than the maximum allowed value.

This numerical method is used to predict the real contact area as a function of the contact force between rough surfaces starting from real shape of the surfaces that come in contact. Then using analytical expressions it is possible to extract the electrical contact resistance. The novel approach relies on a reverse engineering method to generate the real shape of the surface. The post-processing generates the distribution of the contact pressure on the contact surface. From the pressure distribution and size of each contact spot it is possible then to use an analytical expression (see next section) to extract the electrical contact resistance. 

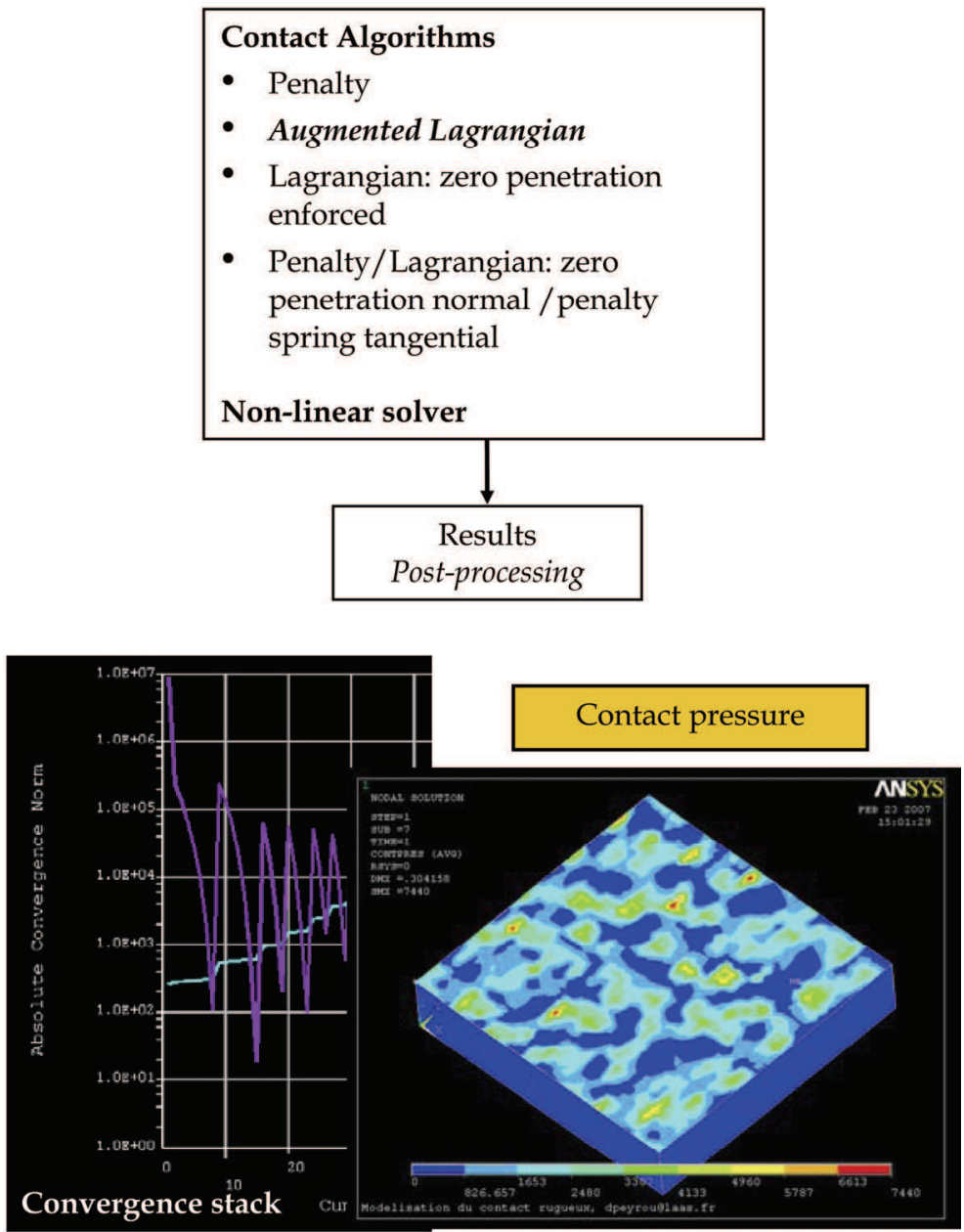

Figure 12. Contact algorithms and post processing in ANSYS

\subsubsection{Overview of theories for the electrical contact resistance}

Several works have already been published about the different theories describing the electrical contact. Thus, this part proposes several ways to estimate the Electrical Contact Resistance (ECR) called Rc, depending at the same time on the mechanical behaviour, directly governing by the contact area, and on electrical assumptions.

Contact resistance models for a single circular contact spot

The way the electrons are transported through electrical connections (ballistic, quasi-ballistic or ohmic transport) between two contact parts of a MEMS switch needs to be determined in order to evaluate the resistance of contact, (Coutu et al., 2006). 


\section{Ohmic contact}

Firstly, if considering a single circular spot of contact, "Ohmic contact" means that the contact size $a$, that is to say its radius, is at least one order of magnitude higher than the mean free path le of the electrons in the material (le $<<$ a). In this case the Ohm's law can be applied everywhere, (Holm, 1999). The measured contact resistance is dominated essentially by a diffuse scattering mechanism, and is given by the Maxwell spreading resistance formula (Eq.3):

$$
R_{\text {Maxwell }}=\frac{\rho}{2 a}
$$

\section{Ballistic contact}

This model must be used if the mean free path of the electrons, $l e$, is high compared with the radius of contact. The conduction of the contact spot is then dominated by a semi-classical approximation called Sharvin's resistance (Sharvin, 1965):

$$
R_{S}=\frac{4 \rho K}{3 \Pi a} \quad \text { where } K=\frac{1_{e}}{a}
$$

where $a$ is the radius of the contact spot.

\section{Mixed conduction}

If the two precedent models are not applicable $\left(l_{\mathrm{e}} \sim \mathrm{a}\right)$, a mixed one has to be used in this middle situation. Wexler (Wexler, 1966) has given a solution of the Boltzmann equation using variational principle to maintain the continuity of the conduction behaviour between the diffusive and the ballistic domain:

$$
\mathrm{R}_{\mathrm{W}}=\mathrm{R}_{\mathrm{S}}+\Gamma(\mathrm{K}) \mathrm{R}_{\mathrm{M}} \text { where } \Gamma(\mathrm{K})=\frac{2}{\Pi} \int_{0}^{\infty} \mathrm{e}^{-\mathrm{Kx}} \operatorname{Sinc}(\mathrm{x}) \mathrm{dx}
$$

with $\Gamma(\mathrm{K})$ a slowly varying Gamma function. Mikrajuddin et al., 1999, derived a well behaved Gamma function. In conclusion, the only criterion used here to discriminate the models is the radius of the contact area $a$ compared to the mean free path of the electrons in the material le.

Secondly, it is important to keep in mind that, generally, the current flows is spread over multiple asperities. Three different models with the consideration of interactions between each contact point can be used to find approximate solutions: Holm's, Greenwood's and Boyer's models.

\section{Contact resistance models for a cluster of circular microcontact}

In general, multiple asperities come into contact resulting in multiple contact spots of varying sizes. The effective contact resistance arising from the contact spots depends on the radii of the spots and the distribution of the spots on the contact surface.

\section{Ballistic contact}

This model can be correctly used if the radius of the apparent contact area (that contains all the contact spots) is smaller than the electron mean free path. In this case, the formula of Sharvin's resistance is applied by computing the resistance of a circular area of radius aeff 
and of area equal to the total area of all the individual contact spots combined (for $\mathrm{N}$ asperities, aeff $=$ N.a).

\section{Ohmic contact}

A lower bound can be obtained on the contact resistance by assuming that contact spots are independent and conduct in parallel. Exact solution when the radii of contact spots are small compared to the separation between the spots. Denoting the contact resistance of a spot $i$ as Ri:

$$
\frac{1}{R_{\mathrm{lb}}}=\sum_{\mathrm{i}} \frac{1}{\mathrm{R}_{\mathrm{i}}}
$$

Considering the case of $n$ elementary spots of radius $a$ regularly spread in a disc of radius $\mathrm{R}$ representing the interface of contact of two metals of equal resistivity $\rho$, an improved expression) of the resistance is proposed, (Timsit, 1999), (Holm, 1999) :

$$
\mathrm{R}_{\mathrm{Holm}}=\frac{\rho}{2 \mathrm{na}}+\frac{\rho}{2 \mathrm{R}}
$$

where the first term represents the resistance of all the spots in parallel, and the second term, the resistance due to the interaction between all the spots.

Greenwood (Greenwood, 1966) derived a formula for the constriction resistance of a set of circular spots. The electrodes communicate via the spots with no interface film between them.

$$
\mathrm{R}_{\mathrm{GW} 1}=\frac{\rho}{2 \sum \mathrm{a}_{\mathrm{i}}}+\frac{\rho}{\Pi} \times \frac{\sum \sum_{\mathrm{i}^{j} \mathrm{j}} \frac{\mathrm{a}_{\mathrm{i}} \mathrm{a}_{\mathrm{i}}}{\mathrm{d}_{\mathrm{ij}}}}{\left(\sum \mathrm{a}_{\mathrm{i}}\right)^{2}}
$$

with ai the radius of the spot $i$, dij the distance between the centers of the spots $i$ and $j$. If there is no correlation between the size of a given spot and its position, the author presented a formula resulting from an approximation of equation 8 :

$$
\mathrm{R}_{\mathrm{GW} 2}=\frac{\rho}{2 \sum \mathrm{a}_{\mathrm{i}}}+\frac{\rho}{\pi \mathrm{n}^{2}} \times \sum \sum_{\mathrm{i} j \mathrm{j}} \frac{1}{\mathrm{~d}_{\mathrm{ij}}}
$$

This formula is exact when the $\mathrm{n}$ spots are all the same size.

In the case where the spots are regularly spread inside a disc of radius $\mathrm{R}$, Greenwood substitutes the double summation in (Eq. 9) by $16 n^{2} / 3 \Pi R$, using the Timoshenko and Goodier's approximation :

$$
\mathrm{R}_{\mathrm{GW} 3}=\frac{\rho}{2 \mathrm{Na}}+\frac{16 \rho}{3 \Pi^{2} \mathrm{R}}
$$

And as $16 / 3 \Pi^{2}=0.5404$, the equation 10 is close to the Holm's expression (Eq. 7). 
As this expression is still incorrect with $\mathrm{R}=\mathrm{a}$ and $\mathrm{n}=1$, Boyer, Noël and Houzé (Boyer et al., 1990), (Boyer, 2001) introduce a new expression for the constriction resistance which introduce a correction term that will be effective in the limit case of a single contact spot :

$$
\mathrm{R}_{\text {Boyer }}=\frac{\rho}{2 \mathrm{Na}}+\frac{\rho}{2 \mathrm{R}}\left(1-\frac{1}{\sqrt{\mathrm{N}}}\left(\mathrm{N} \frac{\mathrm{a}^{2}}{\mathrm{R}^{2}}\right)^{\frac{\sqrt{\mathrm{N}}-1}{2}}\right.
$$

In conclusion, the radius of the contact area $a$ and the number of asperities $N$, and its comparison with the mean free path of the electrons in the material le, allow us to discriminate an electric contact model in diffusive or ballistic electron transport. Then, for a cluster of microcontact, the possible presence of interaction between different asperities leads to the choice of the more appropriate model.

\section{Influence of contamination thin films}

When a conductive film of surface resistance $\lambda\left(\Omega \mathrm{m}^{2}\right)$ is present between two electrodes communicating through a circular spot of radius a, a resistance due to the presence of this film Rfilm has to be added to the Maxwell resistance $R_{M}$.

$$
\mathrm{R}_{\text {film }}=\frac{\lambda}{\Pi^{2}}
$$

For $\mathrm{n}$ circular contact spots of radius a, the resistance Rfilm of the interface film is:

$$
\mathrm{R}_{\text {film }}=\frac{\lambda}{\text { nпа }_{\text {eff }}^{2}}
$$

\subsubsection{Results and discussion}

In ANSYS post-processing, we can extract the nodes on the contact surface for which the contact pressure is not null. Next, we developed a program allowing the extraction of the real shape and dimensions of each contact spot. From the distribution and size of the contacts spots we can use an analytical expression (based on Wexler's approximation of the integral) to extract the electrical contact resistance (Eq. 12). In fact, for both ohmic constriction and boundary scattering, the contact resistance Rc for a spot of radius $a$ is, (Wexler, 1966) and (Coutu, 2006) :

$$
\mathrm{R}_{\mathrm{c}}=\Gamma\left(\frac{1_{\mathrm{e}}}{\mathrm{a}}\right) \mathrm{R}_{\mathrm{M}}+\mathrm{R}_{\mathrm{S}}=\frac{1+0.83\left(\frac{\mathrm{l}_{\mathrm{e}}}{\mathrm{a}}\right)}{1+1.33\left(\frac{\mathrm{l}_{\mathrm{e}}}{\mathrm{a}}\right)} \frac{\rho}{2 \mathrm{a}}+\frac{4 \rho \mathrm{l}_{\mathrm{e}}}{3 \Pi \mathrm{a}^{2}}
$$

Where $l_{\mathrm{e}}$ is the electron mean free path, and $\rho$ is the electrical resistivity. RM is the Maxwell spreading resistance (the resistance due to lattice scattering, diffusive transport), and RS is the Sharvin resistance (the additional resistance due to boundary scattering in small constrictions, ballistic transport).

Using this model for a single spot and the Greenwood's model for a cluster of microcontacts (Eq.6), we performed the contact resistance of all single contact spots in parallel and then added the resistance due to their interaction. The graph figure 13 illustrates the evolution of contact resistance as a function of the applied force. 


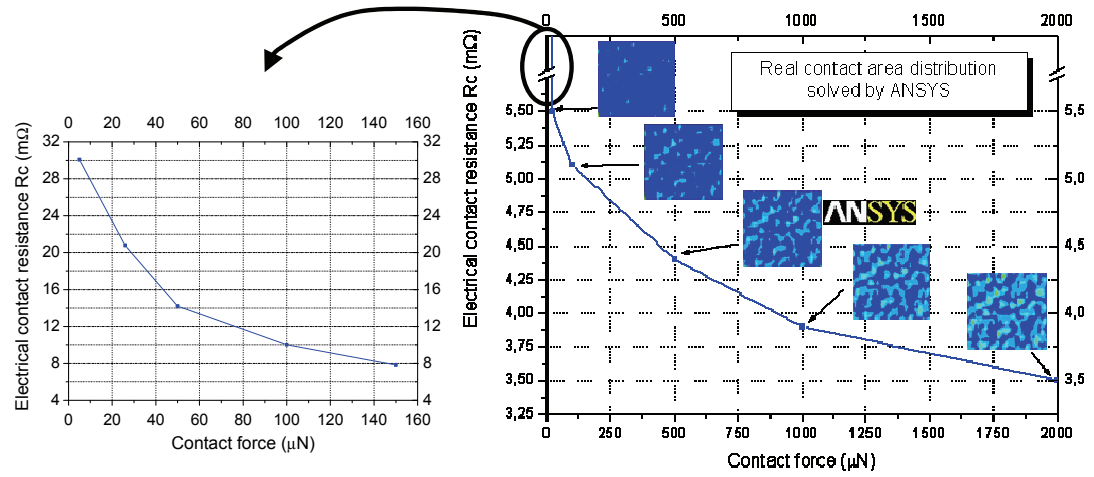

Figure 13. Electrical contact resistance versus contact force

\section{Application manager for interfacing different CAD}

\subsection{Introduction}

To simulate different topologies of deformable micro structures, some multiphysics softwares are under investigation. In order to weigh the software, the criteria are the precision of the results with an acceptable time of calculation to simulate different models with multiple variables. The existance of COMSOL 3.3, at a time linking all the physics and having a good interactive interface seemed to be a good solution. Being incomplete, COMSOL 3.3 needed to be linked to another software to complete its functions and make it more powerful. As a first step, we validated the numerical platform by comparing results of different softwares (Peyrou et al., 2006) and also some analytical solutions (Achkar et al., 2007). The basic advantages and drawbacks of each softwares were considered then we have developed a method to link COMSOL and ANSYS since we think they are complementary. In terms of time of calculation, an interesting result concerning the speed of COMSOL to solve the problem when compared to ANSYS, while keeping the same precision of calculation.

\subsection{Problem definition}

In the domain of structural mechanics, the major difficulty in design relies on, modelling MEMS having high aspect ratio, properly defining the material properties used, and well describing the geometry defined by the technological process.

Generally, in MEMS simulations, we need to combine with the mechanical simulation other physics like Electrostatics, Piezoelectricity... from where comes the need for multi-physics softwares. Multiphysics softwares are now getting more abundant in the research domain specialy in the MEMS domain where different physics find their arena to show their interaction and their influence on these systems.

For any application in MEMS, deformable micro structures using different kinds of actuation with repetitive behaviour are to be simulated in order to study their possible functionning. This functionning is based on designing a mechanical structure deformed piezoelectricaly (Chen et al., 2004), thermomechanicaly, or electrostaticaly, mainly to get into contact with another part, in order to play an electrical function.

To facilitate the task of designers, we need a multiphysics software offering at a time, a well developed solver to reduce the time of calculation and a facility to build parametric models 
(parametric geometry and parametric properties), without loosing too much accuracy on the results.

Following this need, COMSOL 3.3 appeared as software that is capable to do piezoelectric simulations combined to mechanical structure and contact problems, without forgetting thermal and initial stress effects. The problem appears here, when we need to simulate complicated contact problems and where COMSOL solver fails most of the time.

When talking about contact in COMSOL, it is somehow critical and difficult to converge the simulation specially that we have to vary the contact parameters and it's still not sure to converge. The convergence of the contact model using COMSOL failed; one have then to find a way to link both ANSYS and COMSOL in order to use ANSYS contact models. By exchanging the deformed geometry between them and then running the convenient simulation using the appropriate tool, some simulation problems can be solved.

In this chapter, you will learn how to link the software the most efficient for your application in order to obtain a complete tool to analyse your structures. Both the accuracy of COMSOL in deflection and stress, and the reduced calculation time makes it very useful and specially after completing its contact models with ANSYS contact model.

\subsection{Description of the method}

As already stated, we need to link ANSYS and COMSOL in a way to exchange deformed geometry and treat it in the convenient software. This section describes the full method in order to regenerate geometries starting from the deformation obtained from a mechanical simulation.

A very practical way to run simulations on two different softwares, is to pilot it using Matlab. To achieve our purpose, we created in Matlab some functions which make the piloting and the link between the softwares easier. In what follows, the flow chart shows the key steps of the method by using a simple membrane of $200 \times 200 \mu \mathrm{m}^{2}$ subjected to constant pressure and blocked at its corner.

We can begin the cycle from where we want, either from ANSYS or from COMSOL. In the flow chart, we began from COMSOL, with a simple membrane under pressure (Figure 14). This is a typical material characterization test used in MEMS (Xiang \& Vlassak, 2004) and (Xiang \& Vlassak, 2005).

The geometry is drawn, and then the loads and boundary conditions are applied. A mechanical simulation is run in order to obtain the deformation of the membrane. Considering that a contact analysis is needed after the deformation of the membrane, so we export the deformation of the membrane into a text file. In the text file there will be the displacement of each node of the chosen surface of interest as well as some data that we don't need. Matlab will treat this file to clean unneeded information and to reorganize data in the file. The treated file will be used then to create a cloud of keypoints, by creating it one by one, and then generating surfaces from the keypoints. In ANSYS now, we will mesh the regenerated geometry, we will define the physics that we need to study (in our case it's the contact) and then run a new simulation. The deformation is once again output in a text file, giving the displacement value for each node of the surface of interest. Another treatment and organization of the output data file, before being injected in the function that we created in Matlab which regenerates the surface and export it to COMSOL. The geometry is ready to be used in COMSOL, new objects can be added to it, for example electrode, before going into meshing and then boundary condition, to finish into a new simulation. The loop can turn as much times as we need, passing from software to another, until we attain our goal. 

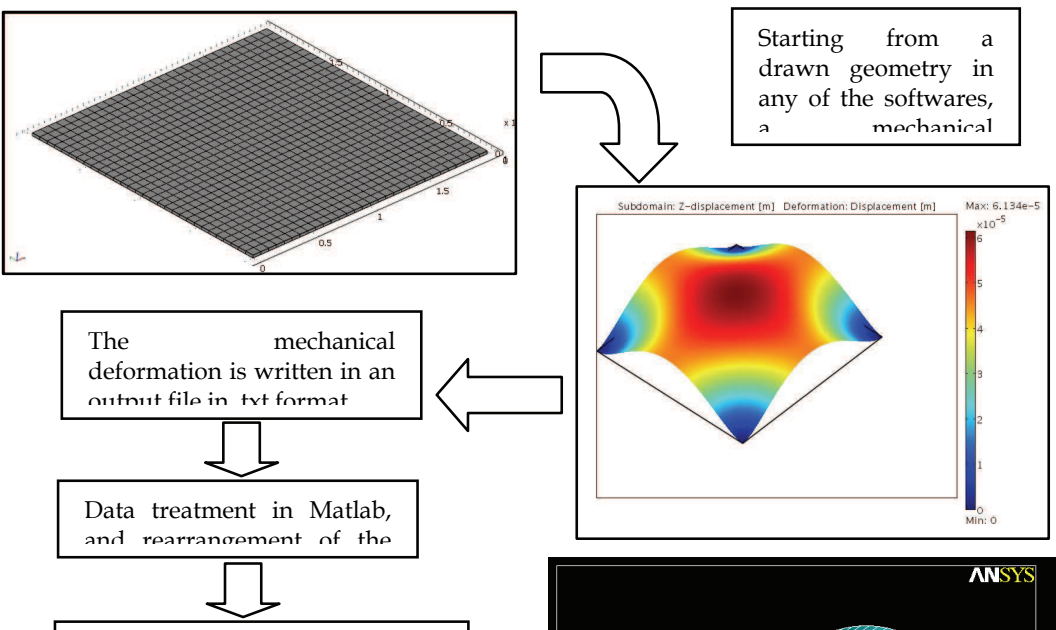

From a set of point cloud, we create surfaces that will form the daformad ranmotru

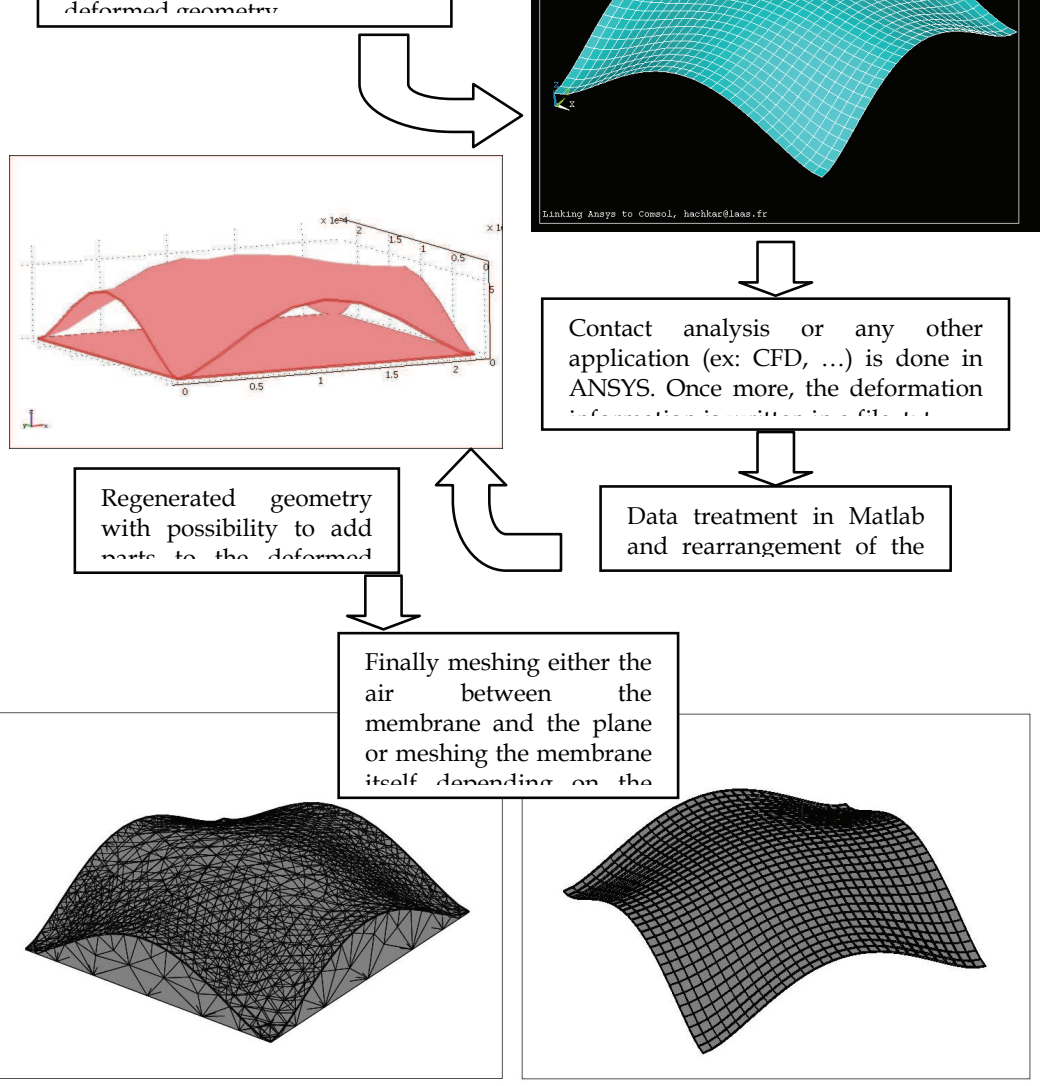

Figure 14. Electrical contact resistance versus contact force 


\section{Acknowledgement}

The authors would like to acknowledge the French National Center for Scientific Research (CNRS) and the University of Toulouse, FRANCE. Also, authors are grateful for the teams' support of the Microdevices and Microsystems of Detection (M2D) Department and the Micro and Nanosystems for wireless Communications (MINC) Department at the Laboratory for Analysis and Architecture of Systems (LAAS) of Toulouse, FRANCE.

\section{References}

Achkar, H.; Pennec, F.; Peyrou, D.; Ahmad, M.AL. Sartor, M.; Plana, R.; Pons, P. (2007). Validation of simulation platform by comparing results and calculation time of different softwares, Proceeding of EUROSIME 2007, (April 2007) pp.520-524

ANSYS : http://www.ansys.com/

Boyer, L.; Noël, S. \& Houzé, F. (1990). Constriction resistance of a multispot contact : an improved analytical expression, 36th IEEE Holm Conf. on Electric Contacts Montreal Canada, (August 1990) pp.134-136

Boyer, L. (2001). Contact Resistance Calculations: Generalizations of Greenswood's Formula Including Interface Films, IEEE Trans. Comp. Pack. Tech., Vol.24, (March 2001) pp.5058

Chen, X; Fox, C H J.; William, S Mc. (2004). Modeling of a tunable capacitor with piezoelectric actuation, J.Micromech. Microeng,Vol.14, (2004) pp.102-107

COMSOL : http://www.comsol.com/

Coutu, R.A.; Reid, J.R.; Cortez, R.; Strawser, R.E.; Kladitis, P.E. (2006) Microswitches with sputtered $\mathrm{Au}, \mathrm{AuPd}, \mathrm{Au}-\mathrm{on}-\mathrm{AuPt}$, and $\mathrm{AuPtCu}$ alloy electric contacts, IEEE Trans. On Components and Packaging Technologies, Vol.29; No.2, (June 2006) pp 341-349

Greenwood, J. A. (1966). Constriction resistance and the real area of contact, Brit. J. Appl. Phys., Vol.17, (1966) pp. 1621-1632

Holm, R. (1969). Electric Contacts: Theory and Applications, Fourth Edition, Berlin, Springer-Verlag, 2000, ISBN-3540038752

Mellé, S.; Conto, D.De; Dubuc, D.; Grenier, K., Vendier, O.; Muraro, J.L.; Cazaux, J.L.; Plana, R. (2005) Reliability Modeling of capacitive RF-MEMS, IEEE Transactions on MicrowaveTheory and Techniques, Vol.53, No.11, (November 2005) pp.3482-3488.

Mikrajuddin, A.; Shi, F.; Kim, H. \& Okuyama, K. (1999). Size-dependent electrical constriction resistance for contacts of arbitrary size : from Sharvin to Holm limits, Proc. Mater. Sci. Sem., Vol. 2, (1999) pp. 321-327

Palasantzas, G. \& DeHosson, J.Th.M. (2006) Surface roughness influence on the pull-in voltage of microswitches in presence of thermal and quantum vacuum fluctuations, Surfaces Sciences, Vol.600, No.7 (April 2006) pp 1450-1455

Peyrou, D. \& al. (2006). Multiphysics Softwares Benchmark on Ansys / Comsol Applied For RF MEMS Switches Packaging Simulations, Eurosime 2006, (April 2006) pp.494-501

Rebeiz, G.M. \& Muldavin, J.B. (2001). RF MEMS switches and switch circuits, IEEE Microwave magazine, pp. 59-71

Rebeiz, G. (2003), RF MEMS Theory, Design and Technology, New Jersey: Wiley, ISBN0471201693. 
Reid, J.R. \& Webster, R.T. (2002) Measurements of charging in capacitive microelectromechanical switches, Electron lett, Vol.38, No24, (November 2002) pp 1544-1545.

Sharvin, Y. V. (1965). Sharvin Resistance Formula, Sov. Phys. JETP, Vol. 21, (1965) p. 655

Sprengen, W.M. van; Puers, R.; Mertens, R. \& Wolf, I.De (2004) A comprehensive model to predict the charging and reliability of RF MEMS switches, J.Micromech. Microeng., Vol.14, No.4, (January 2004) pp 514-521.

Timsit, R.S. (1999). Electrical contact resistance: Fundamental principles, Electrical Contacts: Principles and Applications, Ed. P.G. Slade, (1999) pp.1-88, Marcel Dekker, New York

Wexler, G. (1966). The size effect and the nonlocal Boltzmann transport equation in orifice and disk geometry, Proc. Phys. Soc., Vol.89, (1966) pp. 927-941.

Xiang, Y. \& Vlassak, J.J. (2004). The effects of passivation layer and film thickness on the mechanical behavior of freestanding electroplated $\mathrm{Cu}$ thin films with constant microstructure, Mat. Res. Soc. Symp. Proc., Vol. 795, (2004)

Xiang, Y. \& Vlassak, J.J. (2005). Bauschinger effect in thin metal films, Scripta Materialia, Vol.53, (2005) pp.177-182

Yuan, X.; Peng, Z.; Wang, J.C.M.; Forehand, D.; Goldsmith, C. (2006) Acceleration of dielectric charging in RF MEMS capacitive switches, IEEE Transactions on device and materials reliability, Vol.6, No.4, (December 2006) pp 556-563I 


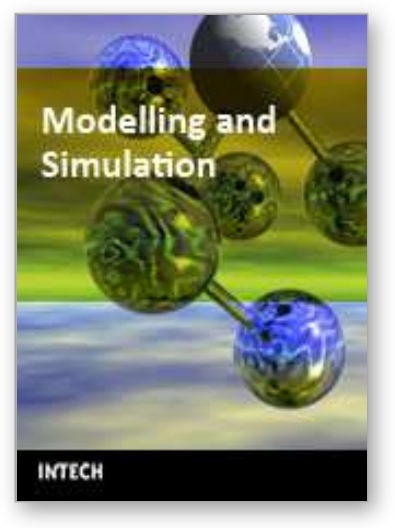

\author{
Modelling and Simulation \\ Edited by Giuseppe Petrone and Giuliano Cammarata
}

ISBN 978-3-902613-25-7

Hard cover, 688 pages

Publisher I-Tech Education and Publishing

Published online 01, June, 2008

Published in print edition June, 2008

This book collects original and innovative research studies concerning modeling and simulation of physical systems in a very wide range of applications, encompassing micro-electro-mechanical systems, measurement instrumentations, catalytic reactors, biomechanical applications, biological and chemical sensors, magnetosensitive materials, silicon photonic devices, electronic devices, optical fibers, electro-microfluidic systems, composite materials, fuel cells, indoor air-conditioning systems, active magnetic levitation systems and more. Some of the most recent numerical techniques, as well as some of the software among the most accurate and sophisticated in treating complex systems, are applied in order to exhaustively contribute in knowledge advances.

\title{
How to reference
}

In order to correctly reference this scholarly work, feel free to copy and paste the following:

Peyrou David, Coccetti Fabio, Achkar Hikmat, Pennec Fabienne, Pons Patrick and Plana Robert (2008). A New Methodology for RF MEMS Simulation, Modelling and Simulation, Giuseppe Petrone and Giuliano Cammarata (Ed.), ISBN: 978-3-902613-25-7, InTech, Available from:

http://www.intechopen.com/books/modelling_and_simulation/a_new_methodology_for_rf_mems_simulation

\section{INTECH}

open science | open minds

\section{InTech Europe}

University Campus STeP Ri

Slavka Krautzeka 83/A

51000 Rijeka, Croatia

Phone: +385 (51) 770447

Fax: +385 (51) 686166

www.intechopen.com

\section{InTech China}

Unit 405, Office Block, Hotel Equatorial Shanghai

No.65, Yan An Road (West), Shanghai, 200040, China

中国上海市延安西路65号上海国际贵都大饭店办公楼 405 单元

Phone: +86-21-62489820

Fax: +86-21-62489821 
(C) 2008 The Author(s). Licensee IntechOpen. This chapter is distributed under the terms of the Creative Commons Attribution-NonCommercialShareAlike-3.0 License, which permits use, distribution and reproduction for non-commercial purposes, provided the original is properly cited and derivative works building on this content are distributed under the same license. 